\title{
Foramen Venoso Órbito Cavernoso: Foramen de Warwick
}

\author{
Cavernous Orbital Venous Foramen: Warwick's Foramen
}

\author{
Claudia Araya'; Juan Silva ${ }^{1,2}$; Miguel Soto ${ }^{2}$; Andrés Salcedo ${ }^{3}$; Victoria Donoso ${ }^{1}$ \& Sebastian Arcos $^{1}$
}

\begin{abstract}
ARAYA, C.; SILVA, J.; SOTO, M.; SALCEDO, A.; DONOSO, V. \& ARCOS, S. Foramen venoso órbito cavernoso: Foramen de Warwick. Int. J. Morphol., 36(4):1480-1484, 2018.
\end{abstract}

RESUMEN: El foramen de Warwick o foramen venoso órbito cavernoso, es un foramen inconstante del ala mayor del esfenoides, situado entre la fisura orbitaria superior y el foramen rotundo. Comunica la órbita con la fosa craneal media y/o con la fosa pterigopalatina y permitiría el paso de la vena oftálmica inferior. La presencia del foramen venoso órbito cavernoso varía entre el 0,38 \% y el 0,74 \%. Se describe con forma redondeada o crescéntica (semilunar), unilateral o bilateral. El objetivo de este trabajo fue evidenciar la presencia y las características de foramen venoso órbito cavernoso en cráneos secos de individuos adultos chilenos de ambos sexos. Se analizaron 138 cráneos de individuos adultos y de ambos sexos, en búsqueda del foramen venoso órbito cavernoso para determinar la frecuencia, localización, forma, tamaño, orientación y distancias con respecto a la fisura orbitaria superior y el foramen rotundo. Los forámenes encontrados fueron fotografiados, explorados y medidos. La presencia del foramen venoso órbito cavernoso fue del $2,17 \%$ de la muestra, encontrándose en forma unilateral $(1,45 \%)$ y bilateral $(0,17 \%)$. Con forma redondeada en 3 casos y semilunar en 1 caso. Con orientación hacia la órbita ( 2 casos) y hacia la fosa pterigopalatina ( 2 casos). También se evidenció que cuando está presente el foramen venoso órbito cavernoso, la separación entre la fisura orbitaria superior y el foramen rotundo es mayor que en su ausencia. Nuestro estudio demuestra la presencia del foramen venoso órbito cavernoso en la población chilena, con una frecuencia más alta que la observada en otras poblaciones. La localización, orientación y formas coinciden con la literatura, pero difiere en el tamaño (en forámenes redondeados). También pudimos determinar que la fisura orbitaria superior y el foramen rotundo tienden a encontrarse más cercanos en ausencia del foramen venoso órbito cavernoso y por lo tanto más distante cuando esta estructura está presente. Este hecho no está descrito en la literatura. Los resultados de este estudio son importantes para la anatomía, oftalmología, traumatología, imagenología, cirugía e identificación humana. Finalmente y en virtud de la TAI, proponemos llamar a este foramen, foramen venoso órbito cavernoso.

PALABRAS CLAVE: Órbita; Fosa craneal media; Canales de la órbita; Foramen de Warwick; Foramen venoso órbito cavernoso; Identificación humana.

\section{INTRODUCCIÓN}

En 1892 Luigi Calori (Fig. 1A), describe un foramen anormal de la fosa craneal media (Fig. 1B) que: "permitiría el paso de una rama de la arteria meníngea media, tal vez la lagrimal". Posteriormente en 1951, Roger Warwick (Fig. 2A) describe una: "hendidura ósea que pareciera puentear la fisura orbitaria superior, creando así un agujero semilunar". Ambas descripciones hacen alusión al foramen de Warwick o foramen venoso órbito cavernoso (FVOC).

El FVOC es descrito como un agujero inconstante del ala mayor del hueso esfenoides (Fig. 2B), con forma redondeada o semilunar (crescéntico), situado entre el extremo inferior de la porción medial de la fisura orbitaria superior (FOS) y el foramen rotundo (FR), comunicando en algunos individuos órbita con fosa craneal media (FCM) y en otros órbita con fosa pterigopalatina (FPP) (Calori, 1892;
Warwick, 1951; Bisaria et al., 1996; Bertelli, 2014; Bertelli \& Regoli, 2014; Macchi et al., 2016; Regoli \& Bertelli, 2017).

Según algunos autores, este foramen permitiría el paso de la vena oftálmica inferior desde órbita hacia el seno cavernoso (Warwick; Bisaria et al.; Bertelli), pero también podría comunicar seno cavernoso con plexo venoso pterigoideo, en este último caso, el FVOC tendría una dirección que evidenciaría una comunicación con FPP (Bertelli; Regoli \& Bertelli).

La alteración de la morfología de los agujeros de la base de cráneo podría afectar el número, el tamaño y el trayecto de estructuras nerviosas y/o vasculares importantes. Dentro de estas alteraciones, el FVOC es considerado una rara variación de la FOS. En esta variación se formaría un puente óseo delgado que dividiría el extremo medial de esta abertura

\footnotetext{
${ }^{1}$ Facultad de Medicina, Universidad San Sebastian, Santiago, Chile.

${ }^{2}$ Departamento de Anatomía y Medicina Legal, Facultad de Medicina, Universidad de Chile, Santiago, Chile.

${ }^{3}$ Facultad de Odontología, Universidad San Sebastian, Santiago, Chile.
} 

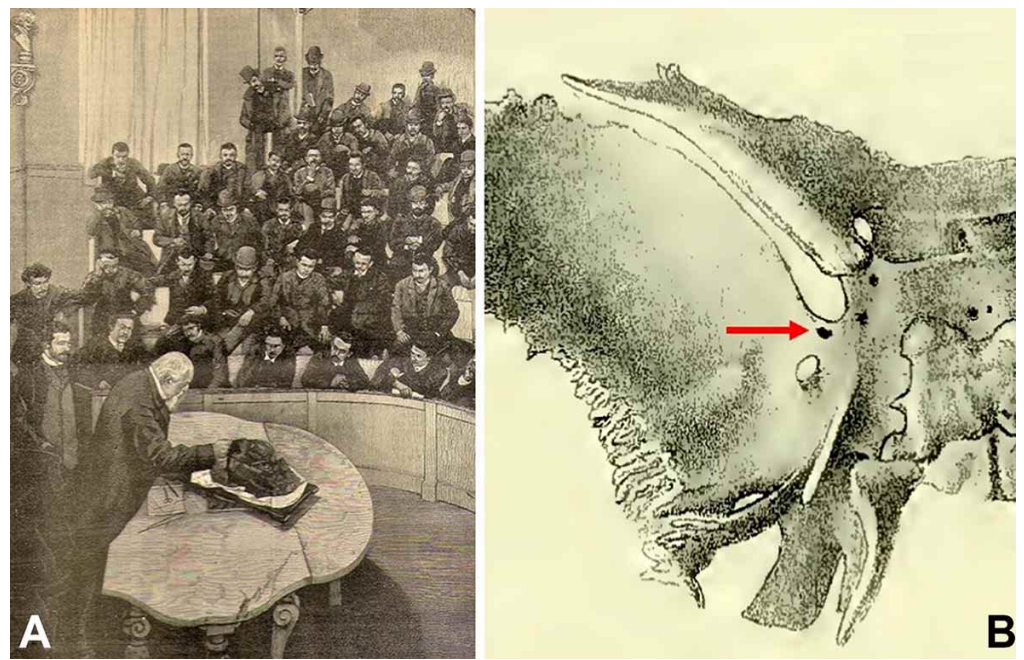

Fig. 1. A. Profesor Luigi Calori (1807-1896), Anatomista de la Universidad de Bolonia (imagen tomada de Ruggeri, 2007) B. Imagen de hueso esfenoides indicando un foramen (flecha), descrito por Calori en 1892, que concuerda con la descripción de Warwick de 1951 (Imagen tomada de Calori, 1892).
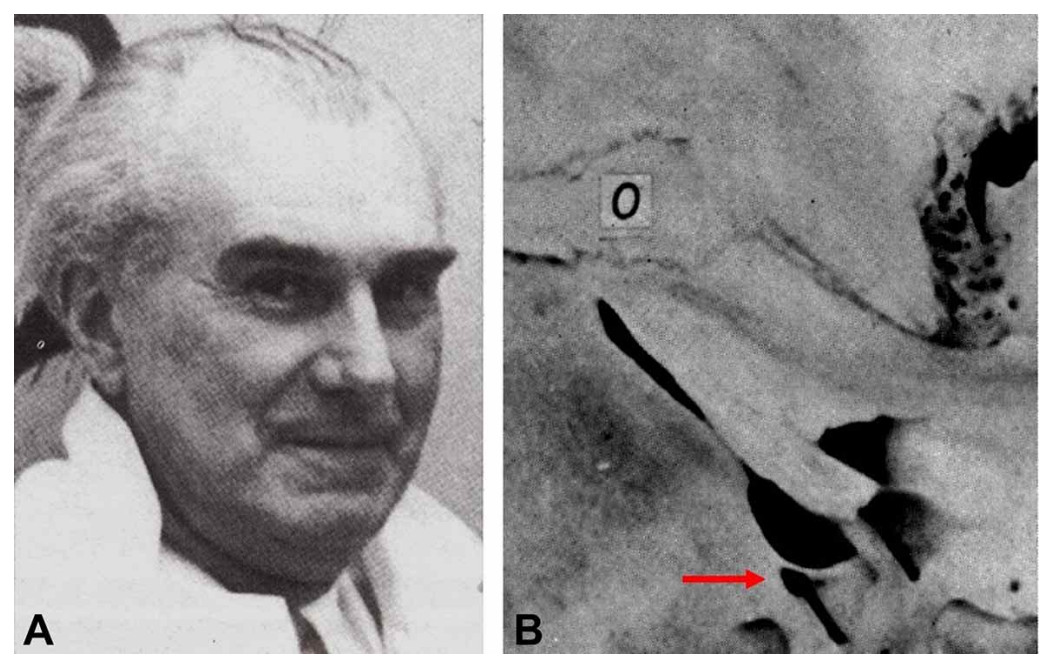

Fig. 2. A. Profesor Roger Warwick (1912-1991), Anatomista de la Universidad de Manchester y del Guy's Hospital (Bowden, 1992). Coeditor de las ediciones 35 a la 37 de Gray's Anatomy (Imagen tomada de Williams, 1998). B. Imagen incluida en Warwick, 1951. En ella se puede observar (flecha) el agujero crescéntico, más tarde conocido como "Foramen de Warwick".

formando un agujero inconstante, bajo este puente (Macchi et al.; Mazengenya \& Ekpo, 2016; Regoli \& Bertelli).

La FOS se describe como una hendidura estrecha que comunica de forma constante, órbita y FCM. En ella convergen el vértice de la órbita, la porción más anterior del seno cavernoso (y su contenido) y entre ambas, la propia FOS (Natori \& Rhoton, 1995). La FOS está formada superiormente por la cara inferior del ala menor del esfenoides y el proceso clinoides anterior. Inferiormente por el margen medial de la superficie orbitaria del ala mayor de este mismo hueso. Ha- cia lateral y arriba, el espacio que separa a ambas alas es cerrado por la porción orbitaria del hueso frontal. Hacia medial completa la fisura, el cuerpo del esfenoides y el puntal óptico (Natori \& Rhoton; Govsa et al., 1999; Regoli \& Bertelli).

La inserción y posición del anillo tendinoso común (ATC) divide a la FOS en 3 compartimentos. Un compartimento lateral, coincidente con la porción estrecha y supero-lateral de la FOS (por fuera del ATC) para los nervios troclear, frontal y lagrimal y a veces la vena oftálmica superior. Un compartimento medial que coincide con el interior del ATC, contiene los ramos superior e inferior del nervio oculomotor, el nervio nasociliar, el nervio abducens, las raíces sensitivas y parasimpáticas del ganglio ciliar y a veces la vena oftálmica superior (este compartimento es llamado agujero oculomotor). Finalmente el compartimento inferior (por dentro y debajo del ATC) es ocupado por tejido adiposo y a veces, la vena oftálmica inferior (Lang \& Kageyama, 1990; Govsa et al.; Reymond et al., 2008; Latarjet \& Ruiz Liard, 2010). Ninguna arteria importante atraviesa por la FOS (Natori \& Rhoton).

La frecuencia observada de FVOC en otros estudios varía entre el 0,38\% (en una muestra de 650 cráneos) y el 0,74\% (en una muestra de 943 cráneos). Principalmente su presencia es unilateral (derecho en el 0,32 $\%$, izquierdo en el $0,42 \%$. En promedio 0,37 $\%)$ y en un solo estudio se observó en forma bilateral (1 individuo representando $0,076 \%)$. Los forámenes de forma redondeada medían $0,98+0,6 \mathrm{~mm}$ y $\operatorname{los}$ forámenes de forma semilunar (crescéntico) midieron 2,88 + 0,95 mm (Bisaria et al.; Bertelli; Regoli \& Bertelli).

El objetivo de este trabajo es evidenciar la presencia de FVOC y describir sus características morfológicas en una muestra de cráneos secos de individuos adultos chilenos de ambos sexos.

El conocimiento detallado de los forámenes propios de la fosa craneal media y sus comunicaciones es importante en anatomía humana normal, en antropología, en oftalmología, en cirugía, en traumatología, en imagenología y en identificación humana. 


\section{MATERIAL Y MÉTODO}

Se analizaron 276 órbitas provenientes de 138 cráneos secos de individuos adultos chilenos de ambos sexos en búsqueda de FVOC. Estos cráneos pertenecen a las osteotecas del Departamento de Anatomía y Medicina Legal de la Facultad de Medicina de la Universidad de Chile y de la Facultad de Medicina de la Universidad San Sebastian.

De los FVOC encontrados, se consideró su localización (unilateral derecho, unilateral izquierdo o bilateral), forma (semilunar o redondeada), tamaño (diámetro vertical y diámetro transversal), distancia entre la FOS y FR, distancia entre la FOS y FVOC, distancia entre el FVOC y FR y la orientación del FVOC (hacia la órbita o hacia la FPP). Por último también se registró la distancia entre FOS y el FR contralateral en aquellos cráneos en que el FVOC es unilateral. Es importante indicar que estas mediciones se realizaron desde FCM.
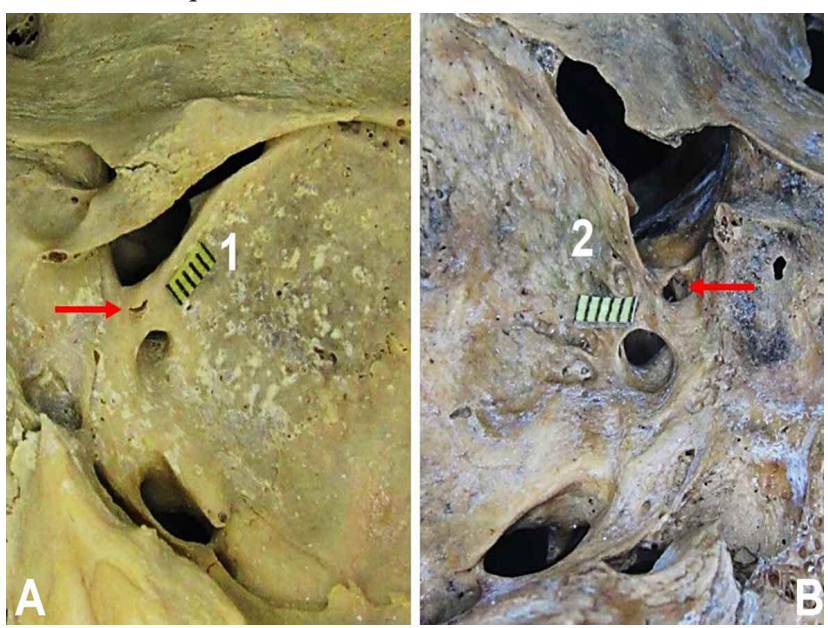

Fig. 3. A. Cráneo individualizado como "1", de sexo masculino de rango etario entre 20 a 30 años, presenta un FVOC de forma de media luna unilateral derecho (flecha). B. Cráneo individualizado como " 2 " de sexo masculino de rango etario entre 60 a 70 años, presenta un FVOC de forma redondeada unilateral izquierdo (flecha). La regleta incluida en las fotografías representa $5 \mathrm{~mm}$.
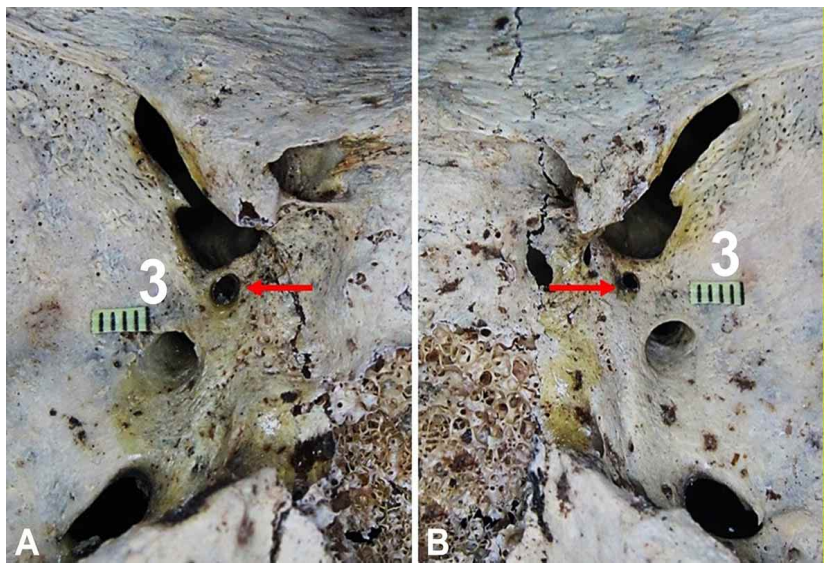

Los cráneos se fotografiaron con cámara digital marca Canon $\odot$ modelo PowerShot $\odot$ D30. Luego se procedió a su medición con compás de precisión marca Staedtler @, modelo Mars 552 y caliper digital marca Mitutoyo@ serie 500 modelo 144. Todas estas mediciones fueron tomadas 3 veces y el valor registrado corresponde al promedio simple. La orientación del FVOC se realizó con sonda metálica. Los resultados se registraron en formulario ad hoc.

\section{RESULTADOS}

Del total de cráneos de la muestra (138), pudimos encontrar 3 individuos que presentaron FVOC, representando el $2,17 \%$. En 2 de ellos el foramen era unilateral (1,45\% de la muestra), correspondiendo a 1 derecho y 1 izquierdo y 1 de ellos era bilateral $(0,17 \%$ de la muestra). De la totalidad de órbitas analizadas (n: 276), pudimos encontrar 4 FVOC, lo que representa el 1,45\% del total de la muestra.

Estos cráneos fueron individualizados y caracterizados de la siguiente manera:

-Cráneo "1", de sexo masculino y rango de edad entre 20 a 30 años, con FVOC unilateral derecho en forma de medialuna, que por su dirección pareciera comunicar FCM con órbita (Fig. $3 \mathrm{~A})$.

-Cráneo "2", de sexo masculino y rango de edad entre 60 a 70 años, con FVOC unilateral izquierdo de forma redondeada, que por su dirección pareciera comunicar FCM con FPP (Fig. 3B).

-Cráneo "3", de sexo masculino y rango de edad entre 40 y 50 años, con FVOC bilateral, ambos de forma redondeada. Por su dirección el izquierdo pareciera comunicar FCM con órbita (Fig. 4A) y el derecho pareciera comunicar FCM con FPP (Fig. 4B). Los resultados de las mediciones realizadas en los FVOC observados, se resumen en la Tabla I.

También pudimos observar, en cráneos con FVOC unilateral, una diferencia importante en la distancia entre FOS y FR versus la separación de estos mismos forámenes en la FCM contralateral. Con FVOC, esta distancia correspondió en promedio a 4,09 mm (rango entre 3,84 y 4,33 $\mathrm{mm}$ ) versus el registro sin FVOC que promedió 2,72 mm (en cráneo " 1 " esta distancia correspondió a 2,46 mm y en cráneo “2”, 2,97 mm).

Fig. 4 A. Cráneo individualizado como "3" de sexo masculino de rango etario entre 40 a 50 años, presenta un FVOC izquierdo de forma redondeada (flecha) B. El mismo cráneo, presenta un FVOC derecho de forma redondeada (flecha). La regleta incluida en las fotografías representa $5 \mathrm{~mm}$. 
Tabla I. La presente tabla resume los resultados en mm, obtenidos en las mediciones realizadas en los FVOC encontrados, además integra los promedios de estas mediciones.

\begin{tabular}{cccccccc}
\hline Cráneo & Forma & Trans & Vert & FOS-FR & FOS-FR contra & FOS-FVOC & FVOC-FR \\
\hline 1 & SL & 1,62 & 1,06 & 3,84 & 2,46 & 1,36 & 1,64 \\
2 & Red & 2,86 & 1,65 & 4,33 & 2,97 & 0,67 & 2,5 \\
3-Der & Red & 1,64 & 1,64 & 5,62 & - & 1,05 & 3,98 \\
3-Izq & Red & 2,54 & 1,92 & 5,51 & - & 0,82 & 3,7 \\
\multicolumn{2}{r}{ Promedio } & & 2,17 & 1,57 & 4,83 & 2,72 & 0,98 \\
\hline
\end{tabular}

SL: foramen de forma semilunar. Red: Foramen de forma redondeada. Trans: dimensión transversal del foramen. Vert: dimensión vertical del foramen. FOS-FR: distancia entre FOS y FR. FOS-FR contra: distancia entre FOS y FR en FCM contralateral. FOS-FVOC: distancia entre FOS y el FVOC. FVOC-FR: distancia entre FVOC y FR.

\section{DISCUSIÓN}

En nuestro estudio pudimos evidenciar la presencia de FVOC en una muestra de cráneos secos de individuos chilenos de ambos sexos tal como está descrito en la literatura (Calori; Warwick; Bisaria et al.; Bertelli; Bertelli \& Regoli; Macchi et al.; Regoli \& Bertelli). La frecuencia de observación fue más alta que aquella determinada en estudios anteriores $(0,38$ al $0,74 \%)$, pudiendo evidenciar que en nuestra muestra el FVOC está presente en un 2,17 \% del total de cráneos (3 de 138) y un 1,45\% del total de órbitas (4 de 276) (Bertelli; Bisaria et al.; Regoli \& Bertelli).

Las formas observadas en los FVOC de la muestra, coincide con lo descrito, pudiendo evidenciar agujeros en forma semilunar (crescéntico) y redondeados (Warwick; Bertelli).

En los forámenes redondeados el diámetro vertical determinado correspondió a 1,74 mm en promedio, con un rango entre 1,64 y 1,92. El diámetro transversal promedió 2,35 mm con un rango entre 1,64 y 2,86. En el foramen semilunar, su diámetro vertical fue de 1,06 y su diámetro horizontal 1,62. Estos resultados son discordantes en referencia al tamaño de los forámenes redondeados $(0,98+0,6 \mathrm{~mm})$ pero son bastante similares en el tamaño del foramen semilunar descrito $(2,88+$ 0,95 mm) (Bisaria et al.; Bertelli; Regoli \& Bertelli).

En concordancia con los autores, pudimos observar el FVOC tanto en forma unilateral ( $1,45 \%$ de la muestra) como bilateral $(0,17 \%)$, pero su frecuencia es más alta a la descrita en la bibliografía (unilateral 0,37 \% y bilateral 0,076\%). En forma coincidente con la literatura pudimos evidenciar el FVOC en dirección hacia la órbita y otros en dirección hacia la fosa pterigopalatina (Bisaria et al.; Bertelli).

Debido a la baja frecuencia en la que se observa FVOC en este estudio y lo descrito en la literatura, no es posible concluir ninguna aseveración de forma fehaciente en relación al sexo y en relación a la frecuencia de unilateralidad y bilateralidad.
A través de este estudio pudimos observar que en aquellos cráneos con FVOC unilaterales, la distancia entre FOS y FR era mayor que la observada en forma contralateral. Estos agujeros tienden a encontrarse más cercanos en ausencia de FVOC (2,72 mm) y por lo tanto más lejanos en aquellos individuos que si presentan esta estructura $(4,83$ $\mathrm{mm})$. Este último hecho no está descrito en la literatura.

Coincidimos con los autores en que este foramen ha sido descrito solo en forma ocasional, que no se conocen bien las estructuras que ocupan este foramen y que solo se puede especular que estaría ocupado por una vena comunicante entre seno cavernoso y órbita o entre seno cavernoso y plexo pterigoideo (Bertelli).

Considerando la importancia de la Terminología Anatómica Internacional, proponemos llamar al foramen de Warwick, foramen venoso órbito cavernoso.

A modo de conclusión, indicaremos que el conocimiento amplio y profundo de los agujeros de la fosa craneal media es vital para las ciencias morfológicas. También es muy importante para el diagnóstico y terapéutica en oftalmología y en traumatología, para el diagnóstico imagenológico de órbita y de base del cráneo, para la correcta planificación del operatorio y postoperatorio quirúrgico y para la identificación humana en medicina legal.

ARAYA, C.; SILVA, J.; SOTO, M.; SALCEDO, A.; DONOSO, V. \& ARCOS, S. Cavernous orbital venous foramen: Warwick's foramen. Int. J. Morphol., 36(4):1480-1484, 2018.

SUMMARY: The Warwick's foramen or cavernous orbital venous foramen, is an inconstant foramen from the greater wing of the sphenoid bone, located between the superior orbital fissure and the rotundum foramen. It connects the orbit with the middle cranial fossa and/or with the pterygopalatine fossa and allows for the 
passage of the inferior ophthalmic vein. The presence of the cavernous orbital venous foramen varies between $0.38 \%$ and 0.74 $\%$ in human skulls. It is described as having a rounded or crescentic (semilunar), unilateral or bilateral shape. The objective of the present work was to demonstrate the presence and characteristics of the cavernous orbital venous foramen in dry skulls of Chilean adult individuals of both sexes. One hundred and thirty-eight adult skulls of both sexes were analyzed in search of the cavernous orbital venous foramen to determine the frequency, location, shape, size, orientation and distances with respect to the superior orbital fissure and the rotund foramen. Found foramina were photographed, explored and measured. The cavernous orbital venous foramen was present in $2.17 \%$ of the sample, and was both unilateral $(1.45 \%)$ and bilateral $(0.17 \%)$. It had a rounded and lunate shape in 3 and 1 cases, respectively. Moreover, it was orientated towards the orbit ( 2 cases) and towards the pterygopalatine fossa ( 2 cases). It was also evidenced that when the cavernous orbital venous foramen is present, the separation between the superior orbital fissure and the rotund foramen is greater than in its absence. Our study demonstrates the presence of the cavernous orbital venous foramen in the Chilean population, with a higher frequency than described previously. The location, orientation and observed forms agree with the literature, but differ in size (in rounded shape foramen). We could also determine that the superior orbital fissure and the rotundum foramen tend to be closer in the absence of the cavernous orbital venous foramen and, therefore, are more distant when this structure is present. This fact is not described in the literature. The results of this study are important for anatomy, ophthalmology, traumatology, imaging, surgery and human identification. Finally, and by virtue of the International Anatomical Terminology (IAT), we propose to call this structure the cavernous orbital venous foramen.

KEY WORDS: Orbit; Middle cranial fossa; Orbit channels; Warwick's foramen; Cavernous orbital venous foramen; Human identification.

\section{REFERENCIAS BIBLIOGRÁFICAS}

Bertelli, E. \& Regoli, M. Branching of the foramen rotundum. A rare variation of the sphenoid. Ital. J. Anat. Embryol., 119(2):148-53, 2014.

Bertelli, E. Metoptic canal, duplication of the optic canal and Warwick's foramen in human orbits. Anat. Sci. Int., 89(1):34-45, 2014.

Bisaria, K. K.; Kumar, N.; Jaiswall, A.; Sharma, P. K.; Mittal, M. \& Bisaria, S. D. An accessory foramen deep in the infraorbital fissure. J. Anat., 189(Pt. 2):461-2, 1996.

Calori, L. Su varie particolarità osteologiche della base del cranio umano. Mem. R. Accademia Sci. Inst. Bologna. 5(2):287-312, 1892.

Govsa, F.; Kayalioglu, G.; Erturk, M. \& Ozgur, T. The superior orbital fissure and its contents. Surg. Radiol. Anat., 21(3):181-5, 1999.

Lang, J. \& Kageyama, I. Clinical anatomy of the blood spaces and blood vessels surrounding the siphon of the internal carotid artery. Acta. Anat. (Basel), 139(4):320-5, 1990.

Latarjet, M. \& Ruiz Liard, A. Anatomía Humana. Buenos Aires, Médica Panamericana, 2010.

Macchi, V.; Regoli, M.; Bracco, S.; Nicoletti, C.; Morra, A.; Porzionato, A.; De Caro, R. \& Bertelli, E. Clinical anatomy of the orbitomeningeal foramina: variational anatomy of the canals connecting the orbit with the cranial cavity. Surg. Radiol. Anat., 38(2):165-77, 2016.

Mazengenya, P. \& Ekpo, O. Unusual foramen in the middle cranial fossae of adult black South African skull specimens. Surg. Radiol. Anat., 39(7):815-8, 2016.

Natori, Y. \& Rhoton, A. L. Jr. Microsurgical anatomy of the superior orbital fissure. Neurosurgery, 36(4):762-75, 1995.

Regoli, M. \& Bertelli, E. The revised anatomy of the canals connecting the orbit with the cranial cavity. Orbit, 36(2):110-7, 2017.

Reymond, J.; Kwiatkowski, J. \& Wysocki, J. Clinical anatomy of the superior orbital fissure and the orbital apex. J. Craniomaxillofac. Surg., 36(6):346-53, 2008.

Warwick, R. A juvenile skull exhibiting duplication of the optic canals and subdivision of the superior orbital fissure. J. Anat., 85(3):289-91, 1951.

Dirección para correspondencia:

Prof. Dra. Claudia A. Araya B.

Académico - Investigador

Facultad de Medicina

Universidad San Sebastián

Campus Los Leones de Providencia

Direction Lota 2465, Edificio F

Santiago

CHILE

Email: claudia.araya@uss.cl

Recibido : 23-06-2018

Aceptado: 07-09-2018 